\title{
Sexual orientation and health behaviors among college students in Italy
}

\author{
Maria Sironi ${ }^{1 *}\left(10\right.$ and Julie Fricke ${ }^{2}$
}

\section{${ }^{*}$ Correspondence:}

m.sironi@ucl.ac.uk

${ }^{1}$ University College London,

Gower Street, London WC1E 6BT, UK

Full list of author information is available at the end of the article

\begin{abstract}
Studies regarding sexual orientation and health behaviors have largely neglected the Italian context. This research utilizes the second wave of the 'Sexual and Emotional Life of Youths' (SELFY) data set to compare the health behaviors of sexual minority (SM) and heterosexual college students in Italy. Results showed that bisexual women were more likely than heterosexual women to smoke both 1-7 and 8+ cigarettes per day, get drunk sometimes and often/very often, and use marijuana and/or ecstasy sometimes and often/very often. Homosexual women were more likely than heterosexual women to smoke 1-7 cigarettes per day, get drunk often/very often, and use marijuana and/ or ecstasy often/very often. Homosexual men were more likely than heterosexual men to smoke $8+$ cigarettes per day, but less likely to get drunk often/very often. Bisexual men were less likely than heterosexual men to participate in physical activity sometimes, often, and very often. Finally, bisexual men were less likely to exercise often than homosexual men. This work highlights that SM college students in Italy, particularly women, are more likely to engage in specific risky health behaviors compared to their heterosexual counterparts. More attention needs to be given to SMs in Italy to inform the Italian national health care system and to ensure that the appropriate care can be provided when required.
\end{abstract}

Keywords: Sexual orientation, Health, Health behavior, Italy, University, SELFY

\section{Introduction}

It is well established in the literature that sexual minorities (SMs) often report poorer health than their heterosexual counterparts, which includes mental and physical health and increased rates of negative health behaviors, such as smoking, drug use, and binge-drinking (Frost et al., 2015; Lindley et al., 2012; Meyer \& Northridge, 2007; VanKim et al., 2017). Health disparities between SMs and heterosexuals are commonly explained by the minority stress model (Meyer, 2003), which proposes that SMs experience unique and chronic stressors related to their disadvantaged status in society, which may negatively influence their health. To cope with negative states and to avoid social distress, SM individuals may turn to maladaptive behaviors with harmful consequences (Eisenberg \& Wechsler, 2003; Kashubeck-West \& Szymanski, 2008; Mereish, 2014; Newcomb et al., 2012). There is substantial evidence that behaviors, such as smoking, drug use, lack of physical exercise, and heavy alcohol consumption

C The Author(s) 2021. Open Access This article is licensed under a Creative Commons Attribution 4.0 International License, which permits use, sharing, adaptation, distribution and reproduction in any medium or format, as long as you give appropriate credit to the original author(s) and the source, provide a link to the Creative Commons licence, and indicate if changes were made. The images or other third party material in this article are included in the article's Creative Commons licence, unless indicated otherwise in a credit line to the material. If material is not included in the article's Creative Commons licence and your intended use is not permitted by statutory regulation or exceeds the permitted use, you will need to obtain permission directly from the copyright holder. To view a copy of this licence, visit http:// creativecommons.org/licenses/by/4.0/. 
are associated with stress and negative mood states like depression and anxiety (Biddle \& Mutrie, 2008; Kassel et al., 2003; Regier et al., 1990). Notably, college students specifically constitute a vulnerable group due to their developmental stage in life, the nature of adjustment to a new environment in college, academic expectations, and the higher education program (Ibrahim et al., 2013).

It is also necessary to recognize that it is often bisexual individuals that experience poorer health across several domains (Mereish et al., 2017b); as such, results from studies that group SM categories together may be misleading. Bisexual individuals may encounter unique minority stressors (Worthen, 2013), such as biphobia (Bostwick \& Hequembourg, 2014), from both heterosexual and SM communities (Mereish et al., 2017a). The impact of widespread bi-negativity and the potential lack of support from the SM community that often stigmatizes bisexual identities (Mohr \& Daly, 2008) has been argued to encourage bisexual individuals to embrace risk behaviors to a greater extent than lesbian and gay individuals (D'Augelli et al., 2005). Further, disparities often vary by gender (Conron et al., 2010; Hsieh \& Ruther, 2016), so stratifying analyses by men and women is preferred.

Studies that have examined sexual orientation disparities in health behaviors have largely neglected the Italian context (in part due to data limitations). Although Italian society is now more tolerant of SMs than it was a decade ago, the Italian context is very different from the American or European setting (Santona \& Tognasso, 2018). Italy is unique because of the presence of the Vatican State. The Vatican State had a strong influence on Italian development of moral and ethical values, including family choices. Researchers have linked Italy's strong Catholic values to the delayed diffusion of new family behaviors, such as cohabitation, divorce, and non-marital childbearing (De Rose et al., 2008; Vignoli \& Salvini, 2014). Notably, family patterns in Italy have become less rigid overtime and the importance of Catholic prescripts has arguably lessened (Vignoli \& Salvini, 2014). From the Catholic Church's perspective, SMs are seen as a threat to the cultural institution of the family (Salvati et al., 2018). The indifference of the State and the overt interference of the Vatican into "public affairs" justify and reinforce the invisibility of SMs, and (indirectly) the discrimination and violence against them (Worthen et al., 2017). Although progress has been made, Italy remains the only major country in the Western world with a marriage ban for samesex couples.

Ultimately, the religious and political traditions in Italian society maintain a level of social homophobia and homonegativity (Baiocco et al., 2015a, 2015b; Lingiardi et al., 2016), which often leads to the discrimination, stigmatization and victimization of this population (Lingiardi et al., 2005). For example, among a random sample of 1000 lesbian, gay men, and bisexual women and men in Naples, Italy in 2011, 28.3\% and $11.9 \%$ self-reported at least one episode of victimization because of their sexual orientation in their lifetime and in the last year, respectively (Pelullo et al., 2013). Traditional gender differences and gender ideologies also play a role in the homonegative attitudes in Italy (Baiocco et al., 2015a, 2015b). In Italy, traditional gender ideology is widespread and extremely closely related to the concept of machismo, which may be considered as over-conformity to the traditional male gender role (Baiocco et al., 2013; Petruccelli et al., 2015). Sexism is also very common (Salvati et al., 2018). Right-wing political 
conservatism and strong religious and traditional beliefs have been found to cause Italian parents to have negative reactions to a child's coming out (Baiocco et al., 2015a, 2015b).

Considering the Italian context as a whole and the clear presence of minority stressors, SMs in Italy are likely to have a high probability of adopting risk behaviors (Lingiardi et al., 2012), such as smoking, binge-drinking, and physical inactivity, three outcomes commonly associated with SM status. Using the second wave of the 'Sexual and Emotional Life of Youths' (SELFY) data set, this work aims to shed light on the health behaviors of SM college students in Italy (lesbians, bisexuals, and gays) by comparing their smoking, drinking, drug use, and physical activity patterns to their heterosexual counterparts. In line with the theories of minority stress, we hypothesize that SMs will be more likely to have a higher smoking and drinking prevalence and participate in less physical activity than heterosexuals. We also hypothesize, based on previous literature, that bisexuals will smoke more, drink more, and be less physically active than lesbians, gays, and heterosexuals. More attention needs to be given to this potential at-risk population to inform the Italian national health care system and to ensure that the appropriate care can be provided when required.

\section{Data and methods}

This research utilizes the second wave of the 'Sexual and Emotional Life of Youths' (SELFY) data set. ${ }^{1}$ SELFY is a survey that interviews students in their first and second year of Statistics and Economics courses at Italian State Universities. It is coordinated by researchers from the universities of Florence, Messina, and Padua (Caltabiano et al., 2020). The first round was conducted during the 2000-2001 academic year, while the second round was conducted during the first half of 2017 in 28 Italian universities. SELFY combines information on the socioeconomic and demographic background of the students, with data on religiousness, time use, friendships, sexual experiences, romantic relationships, attitudes, and most importantly-for the purpose of this analysis-sexual identity and health behaviors. About 20 researchers are now analyzing the data across Europe. For more detailed information on the description of the survey, data collection and the representativeness of SELFY compared to other Italian data it is possible to refer to the Appendix of Minello et al. (2020).

The 2017 sample initially included 7842 individuals; however, 120 students did not answer the question on sexual identity and were excluded from the analysis. Respondents who did not have information on the health behavior variables or control variables were also excluded, resulting in a final sample that consisted of 6204 individuals: 3222 men and 2982 women.

\section{Outcome variables}

Four different outcome variables were used in the analysis of health behaviors: smoking, drinking, drug use (specifically marijuana and/or ecstasy), and physical activity. Smoking is based on the current number of cigarettes respondents report to smoke per day,

\footnotetext{
1 The SELFY data set is run by a research group of the same name headed by Marcantonio Caltabiano (University of Messina), Gianpiero Dalla-Zuanna (University of Padova), Alessandra Minello (University of Florence), and Daniele Vignoli (University of Florence).
} 
and a categorical variable was created with the following categories: 'never', '1-7 cigarettes/day', or ' 8 or more cigarettes/day'. Drinking is based on how often the respondents declare to 'get drunk', and it is a categorical variable with the following categories: 'never', 'sometimes', or 'often/very often'. Drug use is based on how often respondents smoke marijuana and/or use ecstasy, and a categorical variable was created with the following categories: 'never,' 'sometimes', or 'often/very often'. Finally, physical activity is a categorical variable based on how often the respondents do any sort of sports with the following categories: 'never,' 'sometimes', 'often', and 'very often'.

\section{Independent variable}

In the first round of the SELFY data collection in 2000-2001, only information on sexual behavior was collected, but in the second round of data collection in 2017-2018, students were also asked about their sexual attraction and sexual identity. For this analysis, sexual identity is used (a construct of sexual orientation), which is a categorical variable based on how the respondents chose to identify out of three categories: 'homosexual/ gay/lesbian', 'bisexual', or 'heterosexual'.

\section{Confounders}

The control variables included in the analysis are the age at the interview date (continuous: takes into account both year and month of birth), parental education level (categorical: less than high school, high school, college degree or more), work frequency while studying (categorical: never, sometimes, regularly), relationship status (dichotomous: equal to 1 if they are in a relationship, 0 otherwise), friend approval of sex between persons of the same sex (both categorical: no, somewhat, quite/yes), and parental approval of sex between persons of the same sex (both categorical: no, somewhat, quite/yes).

\section{Statistical analysis}

Because of the documented gender differences in health behaviors, all of the analyses were performed separately for men and women. Students in their first 2 years of Economics and Statistics in Italian public universities, who were interviewed during classes (i.e., attending students), are not representative of Italian college students. Hence, the descriptive statistics of the included variables were computed using probability weights to adjust the sampling design. Following descriptive statistics, multivariate multinomial logistic regressions were run to estimate the association between sexual identity and the outcome variables of smoking, drinking, drug use, and physical activity.

First, the models to examine the association between sexual identity and health behaviors were estimated including only age and the region where the respondents attended university; second, the models were estimated including the control variables in order to determine whether socioeconomic and demographic characteristics or friend and parental approval of sex between same-sex persons could partially explain the relationship between sexual identity and smoking, drinking, drug use, and physical activity.

Since the reference group in the regression models is heterosexuals, the results of those models are unable to identify whether there is a significant difference between homosexuals and bisexuals with regard to health behaviors. As stated above, it is 
often bisexuals that have poorer health across several domains. Hence, we repeated the same analysis using the category 'homosexual' as the reference group.

\section{Results}

The results of the descriptive statistics are reported in Table 1. Respondents primarily identified as heterosexual, and more women than men identified as bisexual (2.9\% and $1.8 \%$, respectively). A significantly higher percentage of men compared to women reported to smoke 8 or more cigarettes per day, as well as get drunk often/ very often, smoke marijuana sometimes and often/very often, and use ecstasy sometimes. However, a significantly higher percentage of men also reported to exercise very often compared to women $(27.1 \%$ and $17.2 \%$, respectively). A significantly higher percentage of women than men reported to be in a relationship, as well as deem religion to be quite/very important. Further, a significantly higher percentage of women than men report that their friends quite/do approve of sex between persons of the same sex ( $42.9 \%$ and $36.8 \%$, respectively). However, for both genders, about $20 \%$ report that their friends do not approve of sex between persons of the same sex, and about $40 \%$ report that their parents do not approve. There was no difference between women and men regarding parental approval of sex between persons of the same sex.

Regarding smoking (Table 2), when controlling for all of the confounders, bisexual women were 2 times more likely to smoke 1-7 cigarettes per day than heterosexual women $(p<0.05)$, as well as 2.7 times more likely to smoke 8 or more cigarettes per day $(p<0.01)$. Similar results were found among homosexual women, as they were 3.3 times more likely than heterosexual women to smoke 1-7 cigarettes per day $(p<0.01)$. Homosexual men were 1.1 times more likely to smoke 8 or more cigarettes per day than heterosexual men $(p<0.01)$.

For drinking (Table 3), when controlling for all of the confounders, bisexual women were 3.5 times more likely than heterosexual women to get drunk sometimes $(p<0.01)$ and 5 times more likely to get drunk often/very often $(p<0.01)$. Homosexual women were 4.9 times more likely than heterosexual women to get drunk often/ very often $(p<0.01)$, while homosexual men were less likely than heterosexual men to get drunk often/very often (OR: $0.9 ; p<0.05)$.

For marijuana and/or ecstasy use (Table 4), when controlling for all of the confounders, bisexual women were 2 times more likely than heterosexual women to use marijuana and/or ecstasy sometimes $(p<0.05)$, and 6.5 times more likely to use them often/very often $(p<0.01)$. Homosexual women were 4.5 times more likely than heterosexual women to use marijuana and/or ecstasy often/very often $(p<0.05)$. Regarding physical activity (Table 5 ), bisexual men were less likely than heterosexual men to participate in physical activity sometimes (OR: $0.4 ; p<0.01$ ), often (OR: 0.7 ; $p<0.01$ ), and very often (OR: $0.2 ; p<0.01$ ). Sexual identity was not significantly associated with physical activity among women.

When the analysis was repeated with changing the reference category to homosexuals, the only significant result was that bisexual men were 0.2 times less likely to exercise often than homosexual men $(p<0.05)$. 
Table 1 Descriptive statistics (weighted)

\begin{tabular}{|c|c|c|}
\hline & Men & Women \\
\hline \multicolumn{3}{|l|}{ Main variables } \\
\hline \multicolumn{3}{|l|}{ Sexual identity (\%) } \\
\hline Heterosexual & 96.7 & 96.3 \\
\hline Bisexual & 1.8 & 2.9 \\
\hline Homosexual & 1.5 & 0.9 \\
\hline \multicolumn{3}{|l|}{ Smoking (\%) } \\
\hline Never & 68.0 & 71.2 \\
\hline $1-7$ cigarettes/day & 17.6 & 19.7 \\
\hline $8+$ cigarettes/day & 14.4 & $91.9^{*}$ \\
\hline \multicolumn{3}{|l|}{ Getting drunk (\%) } \\
\hline Never & 28.0 & $42.4^{*}$ \\
\hline Sometimes & 58.4 & $49.8^{*}$ \\
\hline Often/very often & 13.6 & $7.8^{*}$ \\
\hline \multicolumn{3}{|l|}{ Smoking marijuana (\%) } \\
\hline Never & 58.3 & $71.5^{*}$ \\
\hline Sometimes & 28.4 & $23.8^{*}$ \\
\hline Often/very often & 13.3 & $4.6^{*}$ \\
\hline \multicolumn{3}{|l|}{ Use ecstasy (\%) } \\
\hline Never & 97.1 & $99.0^{*}$ \\
\hline Sometimes & 2.7 & $0.9^{*}$ \\
\hline Often/very often & 0.2 & 0.1 \\
\hline \multicolumn{3}{|l|}{ Physical activity (\%) } \\
\hline Never & 11.3 & $22.4^{*}$ \\
\hline Sometimes & 33.9 & 36.4 \\
\hline Often & 27.6 & 24.0 \\
\hline Very often & 27.1 & $17.2^{*}$ \\
\hline \multicolumn{3}{|l|}{ Control variables } \\
\hline Avg. age & 21.0 & 20.9 \\
\hline \multicolumn{3}{|l|}{ Parents'education (\%) } \\
\hline Less than HS & 10.8 & $17.3^{*}$ \\
\hline High school & 52.2 & 53.4 \\
\hline College degree or more & 37.0 & $29.2^{*}$ \\
\hline \multicolumn{3}{|l|}{ Importance of religion (\%) } \\
\hline Not at all & 32.9 & $20.0^{*}$ \\
\hline Somewhat & 35.1 & 34.5 \\
\hline Quite/very & 32.1 & $45.5^{*}$ \\
\hline \multicolumn{3}{|l|}{ Working while studying (\%) } \\
\hline Never & 49.1 & 52.7 \\
\hline Sometimes & 35.8 & 31.4 \\
\hline Regularly & 15.1 & 15.9 \\
\hline In a relationship (\%) & 38.6 & $51.6^{*}$ \\
\hline \multicolumn{3}{|c|}{ Friends approve sex with persons of same-sex (\%) } \\
\hline No & 23.6 & 22.5 \\
\hline Somewhat & 39.5 & $34.5^{*}$ \\
\hline Quite/yes & 36.8 & $42.9^{*}$ \\
\hline \multicolumn{3}{|c|}{ Parents approve sex with persons of same-sex (\%) } \\
\hline No & 39.4 & 36.1 \\
\hline Somewhat & 39.3 & 40.9 \\
\hline Quite/yes & 21.3 & 23.0 \\
\hline N & 3222 & 2982 \\
\hline
\end{tabular}

*Results are significantly different between men and women $(p<0.05)$ 
Table 2 Multinomial logistic regression, smoking

\begin{tabular}{|c|c|c|c|c|c|c|c|c|}
\hline \multirow{2}{*}{$\begin{array}{l}Y=\text { Smoking (Ref: } \\
\text { Never) }\end{array}$} & \multicolumn{4}{|c|}{ 1-7 cigarettes/day } & \multicolumn{4}{|c|}{$8+$ cigarettes/day } \\
\hline & \multicolumn{2}{|l|}{ Men } & \multicolumn{2}{|l|}{ Women } & \multicolumn{2}{|l|}{ Men } & \multicolumn{2}{|l|}{ Women } \\
\hline \multicolumn{9}{|c|}{ Sexual identity (Ref: Heterosexual) } \\
\hline Bisexual & $\begin{array}{l}0.745 \\
(0.292)\end{array}$ & $\begin{array}{l}0.799 \\
(0.318)\end{array}$ & $\begin{array}{l}2.308^{* * *} \\
(0.668)\end{array}$ & $\begin{array}{l}2.024^{* *} \\
(0.597)\end{array}$ & $\begin{array}{l}0.565 \\
(0.272)\end{array}$ & $\begin{array}{l}0.607 \\
(0.296)\end{array}$ & $\begin{array}{l}3.245^{* * *} \\
(1.101)\end{array}$ & $\begin{array}{l}2.670^{* * *} \\
(0.933)\end{array}$ \\
\hline Homosexual & $\begin{array}{l}0.544 \\
(0.293)\end{array}$ & $\begin{array}{l}0.527 \\
(0.286)\end{array}$ & $\begin{array}{l}3.651^{* * *} \\
(1.577)\end{array}$ & $\begin{array}{l}3.253^{* * *} \\
(1.428)\end{array}$ & $\begin{array}{l}0.625 \\
(0.339)\end{array}$ & $\begin{array}{l}0.644 \\
(0.355)\end{array}$ & $\begin{array}{l}2.670^{*} \\
(1.579)\end{array}$ & $\begin{array}{l}2.326 \\
(1.393)\end{array}$ \\
\hline Age & $\begin{array}{l}1.079^{* *} \\
(0.036)\end{array}$ & $\begin{array}{l}1.063^{*} \\
(0.036)\end{array}$ & $\begin{array}{l}1.052 \\
(0.038)\end{array}$ & $\begin{array}{l}1.038 \\
(0.039)\end{array}$ & $\begin{array}{l}1.146^{* * *} \\
(0.039)\end{array}$ & $\begin{array}{l}1.109^{* * *} \\
(0.039)\end{array}$ & $\begin{array}{l}1.053 \\
(0.052)\end{array}$ & $\begin{array}{l}1.023 \\
(0.052)\end{array}$ \\
\hline \multicolumn{9}{|c|}{ Parents'education (Ref: < then high school) } \\
\hline High school & & $\begin{array}{l}1.535^{* *} \\
(0.264)\end{array}$ & & $\begin{array}{l}1.161 \\
(0.153)\end{array}$ & & $\begin{array}{l}1.196 \\
(0.208)\end{array}$ & & $\begin{array}{l}1.014 \\
(0.188)\end{array}$ \\
\hline College degree & & $\begin{array}{l}1.716^{* * *} \\
(0.307)\end{array}$ & & $\begin{array}{l}1.299^{*} \\
(0.192)\end{array}$ & & $\begin{array}{l}1.438^{* *} \\
(0.263)\end{array}$ & & $\begin{array}{l}1.385 \\
(0.279)\end{array}$ \\
\hline \multicolumn{9}{|c|}{ Importance of religion (Ref: Not at all) } \\
\hline Somewhat & & $\begin{array}{l}0.834 \\
(0.095)\end{array}$ & & $\begin{array}{l}0.886 \\
(0.117)\end{array}$ & & $\begin{array}{l}0.655^{* * *} \\
(0.082)\end{array}$ & & $\begin{array}{l}0.772 \\
(0.140)\end{array}$ \\
\hline Quite/very & & $\begin{array}{l}0.603^{* * *} \\
(0.073)\end{array}$ & & $\begin{array}{l}0.528^{* * *} \\
(0.070)\end{array}$ & & $\begin{array}{l}0.431^{* * *} \\
(0.058)\end{array}$ & & $\begin{array}{l}0.421^{* * *} \\
(0.078)\end{array}$ \\
\hline \multicolumn{9}{|c|}{ Working while in college (Ref: Never) } \\
\hline Sometimes & & $\begin{array}{l}1.410^{* * *} \\
(0.148)\end{array}$ & & $\begin{array}{l}1.417^{* * *} \\
(0.156)\end{array}$ & & $\begin{array}{l}2.118^{* * *} \\
(0.250)\end{array}$ & & $\begin{array}{l}1.702^{* * *} \\
(0.263)\end{array}$ \\
\hline Regularly & & $\begin{array}{l}1.301^{*} \\
(0.193)\end{array}$ & & $\begin{array}{l}1.384^{* *} \\
(0.205)\end{array}$ & & $\begin{array}{l}2.576^{* * *} \\
(0.391)\end{array}$ & & $\begin{array}{l}2.009^{* * *} \\
(0.408)\end{array}$ \\
\hline In a relationship & & $\begin{array}{l}1.11 \\
(0.108)\end{array}$ & & $\begin{array}{l}1.216^{* *} \\
(0.116)\end{array}$ & & $\begin{array}{l}0.892 \\
(0.097)\end{array}$ & & $\begin{array}{l}1.124 \\
(0.151)\end{array}$ \\
\hline \multicolumn{9}{|c|}{ Friends approve sex with persons of same-sex (Ref: No) } \\
\hline Somewhat & & $\begin{array}{l}1.248 \\
(0.171)\end{array}$ & & $\begin{array}{l}1.266^{*} \\
(0.182)\end{array}$ & & $\begin{array}{l}0.97 \\
(0.142)\end{array}$ & & $\begin{array}{l}1.282 \\
(0.258)\end{array}$ \\
\hline Quite/yes & & $\begin{array}{l}1.194 \\
(0.191)\end{array}$ & & $\begin{array}{l}1.334^{*} \\
(0.212)\end{array}$ & & $\begin{array}{l}1.04 \\
(0.176)\end{array}$ & & $\begin{array}{l}1.311 \\
(0.296)\end{array}$ \\
\hline \multicolumn{9}{|c|}{ Parents approve sex with persons of same-sex (Ref: No) } \\
\hline Somewhat & & $\begin{array}{l}0.998 \\
(0.121)\end{array}$ & & $\begin{array}{l}0.843 \\
(0.107)\end{array}$ & & $\begin{array}{l}1.024 \\
(0.136)\end{array}$ & & $\begin{array}{l}0.849 \\
(0.151)\end{array}$ \\
\hline Quite/yes & & $\begin{array}{l}1.121 \\
(0.178)\end{array}$ & & $\begin{array}{l}0.903 \\
(0.144)\end{array}$ & & $\begin{array}{l}0.782 \\
(0.143)\end{array}$ & & $\begin{array}{l}0.748 \\
(0.172)\end{array}$ \\
\hline Constant & $\begin{array}{l}0.071^{* * *} \\
(0.051)\end{array}$ & $\begin{array}{l}0.062^{* * *} \\
(0.048)\end{array}$ & $\begin{array}{l}0.074^{* * *} \\
(0.058)\end{array}$ & $\begin{array}{l}0.101^{* * *} \\
(0.083)\end{array}$ & $\begin{array}{l}0.024^{* * *} \\
(0.018)\end{array}$ & $\begin{array}{l}0.048^{* * *} \\
(0.038)\end{array}$ & $\begin{array}{l}0.073^{* *} \\
(0.076)\end{array}$ & $\begin{array}{l}0.172 \\
(0.190)\end{array}$ \\
\hline N & 3222 & & 2982 & & 3222 & & 2982 & \\
\hline
\end{tabular}

${ }^{*} p<0.10,{ }^{* *} p<0.05,{ }^{* * *} p<0.01$. Region where respondents attend university is included in all models

\section{Discussion}

SMs commonly report poorer health behaviors compared with heterosexuals (Frost et al., 2015; Lindley et al., 2012; Meyer \& Northridge, 2007; VanKim et al., 2017), particularly those who identify as bisexual (Mereish et al., 2017b). Past research regarding SMs has primarily neglected the Italian context (in part due to data limitations). Using the second wave of the SELFY data set, this work aimed to shed light on the health behaviors of SM college students in Italy (lesbians, bisexuals, and gays) by comparing their smoking, drinking, drug use, and physical activity patterns to those of their heterosexual counterparts. In line with the theories of minority stress (Meyer, 2003), we hypothesized that SMs will be more likely to have a higher smoking and drinking prevalence 
Table 3 Multinomial logistic regression, drinking

\begin{tabular}{|c|c|c|c|c|c|c|c|c|}
\hline \multirow[t]{2}{*}{$Y=$ Drinking (Ref: Never) } & \multicolumn{4}{|c|}{ Sometimes } & \multicolumn{4}{|c|}{ Often/very often } \\
\hline & \multicolumn{2}{|l|}{ Men } & \multicolumn{2}{|l|}{ Women } & \multicolumn{2}{|l|}{ Men } & \multicolumn{2}{|l|}{ Women } \\
\hline \multicolumn{9}{|c|}{ Sexual identity (Ref: heterosexual) } \\
\hline Bisexual & $\begin{array}{l}0.709 \\
(0.219)\end{array}$ & $\begin{array}{l}0.799 \\
(0.254)\end{array}$ & $\begin{array}{l}4.186^{* * *} \\
(1.479)\end{array}$ & $\begin{array}{l}3.543^{* * *} \\
(1.275)\end{array}$ & $\begin{array}{l}0.936 \\
(0.405)\end{array}$ & $\begin{array}{l}1.044 \\
(0.464)\end{array}$ & $\begin{array}{l}6.749^{* * * *} \\
(3.098)\end{array}$ & $\begin{array}{l}5.046^{* * *} \\
(2.379)\end{array}$ \\
\hline Homosexual & $\begin{array}{l}0.843 \\
(0.320)\end{array}$ & $\begin{array}{l}0.845 \\
(0.328)\end{array}$ & $\begin{array}{l}1.628 \\
(0.771)\end{array}$ & $\begin{array}{l}1.434 \\
(0.695)\end{array}$ & $\begin{array}{l}1.085 \\
(0.561)\end{array}$ & $\begin{array}{l}1.075 \\
(0.573)\end{array}$ & $\begin{array}{l}5.556^{* * *} \\
(3.166)\end{array}$ & $\begin{array}{l}4.858^{* * *} \\
(2.833)\end{array}$ \\
\hline Age & $\begin{array}{l}1.029 \\
(0.030)\end{array}$ & $\begin{array}{l}1.001 \\
(0.030)\end{array}$ & $\begin{array}{l}0.999 \\
(0.030)\end{array}$ & $\begin{array}{l}0.982 \\
(0.031)\end{array}$ & $\begin{array}{l}0.930^{*} \\
(0.041)\end{array}$ & $\begin{array}{l}0.903^{* *} \\
(0.041)\end{array}$ & $\begin{array}{l}0.941 \\
(0.057)\end{array}$ & $\begin{array}{l}0.929 \\
(0.058)\end{array}$ \\
\hline \multicolumn{9}{|c|}{ Parents' education (Ref: < then high school) } \\
\hline High school & & $\begin{array}{l}1.309^{* *} \\
(0.171)\end{array}$ & & $\begin{array}{l}1.087 \\
(0.116)\end{array}$ & & $\begin{array}{l}1.481^{*} \\
(0.306)\end{array}$ & & $\begin{array}{l}1.266 \\
(0.285)\end{array}$ \\
\hline College degree & & $\begin{array}{l}1.421^{* *} \\
(0.198)\end{array}$ & & $\begin{array}{l}1.401^{* * *} \\
(0.170)\end{array}$ & & $\begin{array}{l}1.981^{* * *} \\
(0.425)\end{array}$ & & $\begin{array}{l}2.253^{* * *} \\
(0.542)\end{array}$ \\
\hline \multicolumn{9}{|c|}{ Importance of religion (Ref: not at all) } \\
\hline Somewhat & & $\begin{array}{l}0.875 \\
(0.096)\end{array}$ & & $\begin{array}{l}1.121 \\
(0.136)\end{array}$ & & $\begin{array}{l}0.542^{* * *} \\
(0.080)\end{array}$ & & $\begin{array}{l}0.97 \\
(0.195)\end{array}$ \\
\hline Quite/very & & $\begin{array}{l}0.446^{* * *} \\
(0.048)\end{array}$ & & $\begin{array}{l}0.605^{* * *} \\
(0.070)\end{array}$ & & $\begin{array}{l}0.285^{* * *} \\
(0.043)\end{array}$ & & $\begin{array}{l}0.321^{* * *} \\
(0.068)\end{array}$ \\
\hline \multicolumn{9}{|c|}{ Working while in college (Ref: Never) } \\
\hline Sometimes & & $\begin{array}{l}1.507^{* * *} \\
(0.141)\end{array}$ & & $\begin{array}{l}1.442^{* * *} \\
(0.135)\end{array}$ & & $\begin{array}{l}2.069^{* * * *} \\
(0.274)\end{array}$ & & $\begin{array}{l}1.640^{* * *} \\
(0.290)\end{array}$ \\
\hline Regularly & & $\begin{array}{l}1.690^{* * *} \\
(0.227)\end{array}$ & & $\begin{array}{l}1.531^{* * *} \\
(0.195)\end{array}$ & & $\begin{array}{l}2.083^{* * *} \\
(0.389)\end{array}$ & & $\begin{array}{l}1.866^{* * *} \\
(0.444)\end{array}$ \\
\hline In a relationship & & $\begin{array}{l}0.91 \\
(0.078)\end{array}$ & & $\begin{array}{l}1.174^{* *} \\
(0.093)\end{array}$ & & $\begin{array}{l}0.714^{* * *} \\
(0.089)\end{array}$ & & $\begin{array}{l}0.878 \\
(0.134)\end{array}$ \\
\hline \multicolumn{9}{|c|}{ Friends approve sex with persons of same-sex (Ref: No) } \\
\hline Somewhat & & $\begin{array}{l}1.219^{*} \\
(0.141)\end{array}$ & & $\begin{array}{l}1.159 \\
(0.134)\end{array}$ & & $\begin{array}{l}1.141 \\
(0.190)\end{array}$ & & $\begin{array}{l}1.272 \\
(0.306)\end{array}$ \\
\hline Quite/yes & & $\begin{array}{l}1.143 \\
(0.156)\end{array}$ & & $\begin{array}{l}1.585^{* * *} \\
(0.207)\end{array}$ & & $\begin{array}{l}1.066 \\
(0.209)\end{array}$ & & $\begin{array}{l}1.800^{* *} \\
(0.469)\end{array}$ \\
\hline \multicolumn{9}{|c|}{ Parents approve sex with persons of same-sex (Ref: No) } \\
\hline Somewhat & & $\begin{array}{l}1.169 \\
(0.125)\end{array}$ & & $\begin{array}{l}1.119 \\
(0.118)\end{array}$ & & $\begin{array}{l}0.924 \\
(0.142)\end{array}$ & & $\begin{array}{l}1.152 \\
(0.239)\end{array}$ \\
\hline Quite/yes & & $\begin{array}{l}0.913 \\
(0.131)\end{array}$ & & $\begin{array}{l}0.991 \\
(0.136)\end{array}$ & & $\begin{array}{l}0.949 \\
(0.190)\end{array}$ & & $\begin{array}{l}1.203 \\
(0.306)\end{array}$ \\
\hline Constant & $\begin{array}{l}1.386 \\
(0.877)\end{array}$ & $\begin{array}{l}2.282 \\
(1.554)\end{array}$ & $\begin{array}{l}1.03 \\
(0.660)\end{array}$ & $\begin{array}{l}1.233 \\
(0.846)\end{array}$ & $\begin{array}{l}1.775 \\
(1.701)\end{array}$ & $\begin{array}{l}3.874 \\
(3.940)\end{array}$ & $\begin{array}{l}0.602 \\
(0.768)\end{array}$ & $\begin{array}{l}0.806 \\
(1.097)\end{array}$ \\
\hline N & 3222 & & 2982 & & 3222 & & 2982 & \\
\hline
\end{tabular}

${ }^{*} p<0.10,{ }^{* *} p<0.05,{ }^{* * *} p<0.01$. Region where respondents attend university is included in all models

and participate in less physical activity than heterosexuals. We also hypothesized that bisexuals will smoke more, drink more, and be less physically active than lesbians, gays, and heterosexuals.

For women and smoking, bisexual women were more likely to smoke 1-7 cigarettes per day than heterosexual women, as well as more likely to smoke 8 or more cigarettes per day. Similar results were found among homosexual women, as they were more likely than heterosexual women to smoke 1-7 cigarettes per day. For men, homosexual men were more likely to smoke 8 or more cigarettes per day than heterosexual men, but no significant differences were found between bisexual and heterosexual men. As such, the hypothesis that homosexual men and women would have a higher smoking prevalence than their heterosexual counterparts was confirmed, 
Table 4 Multinomial logistic regression, marijuana and/or ecstasy use

\begin{tabular}{|c|c|c|c|c|c|c|c|c|}
\hline \multirow[t]{2}{*}{$Y=$ Drugs (Ref: Never) } & \multicolumn{4}{|c|}{ Sometimes } & \multicolumn{4}{|c|}{ Often/very often } \\
\hline & \multicolumn{2}{|l|}{ Men } & \multicolumn{2}{|l|}{ Women } & \multicolumn{2}{|l|}{ Men } & \multicolumn{2}{|l|}{ Women } \\
\hline \multicolumn{9}{|c|}{ Sexual identity (Ref: heterosexual) } \\
\hline Bisexual & $\begin{array}{l}1.079 \\
(0.322)\end{array}$ & $\begin{array}{l}1.198 \\
(0.374)\end{array}$ & $\begin{array}{l}2.287^{* * *} \\
(0.663)\end{array}$ & $\begin{array}{l}1.950^{* *} \\
(0.586)\end{array}$ & $\begin{array}{l}0.407 \\
(0.247)\end{array}$ & $\begin{array}{l}0.443 \\
(0.276)\end{array}$ & $\begin{array}{l}9.449^{* * *} \\
(3.278)\end{array}$ & $\begin{array}{l}6.548^{* * *} \\
(2.466)\end{array}$ \\
\hline Homosexual & $\begin{array}{l}0.995 \\
(0.373)\end{array}$ & $\begin{array}{l}0.9 \\
(0.344)\end{array}$ & $\begin{array}{l}1.833 \\
(0.826)\end{array}$ & $\begin{array}{l}1.595 \\
(0.731)\end{array}$ & $\begin{array}{l}0.728 \\
(0.401)\end{array}$ & $\begin{array}{l}0.653 \\
(0.368)\end{array}$ & $\begin{array}{l}5.812^{* * *} \\
(3.411)\end{array}$ & $\begin{array}{l}4.475^{* *} \\
(2.754)\end{array}$ \\
\hline Age & $\begin{array}{l}1.038 \\
(0.030)\end{array}$ & $\begin{array}{l}1.015 \\
(0.030)\end{array}$ & $\begin{array}{l}1.085^{* *} \\
(0.037)\end{array}$ & $\begin{array}{l}1.080^{* *} \\
(0.038)\end{array}$ & $\begin{array}{l}1.001 \\
(0.038)\end{array}$ & $\begin{array}{l}0.955 \\
(0.038)\end{array}$ & $\begin{array}{l}1.022 \\
(0.074)\end{array}$ & $\begin{array}{l}0.994 \\
(0.076)\end{array}$ \\
\hline \multicolumn{9}{|c|}{ Parents' education (Ref: < then high school) } \\
\hline High school & & $\begin{array}{l}1.552^{* * *} \\
(0.221)\end{array}$ & & $\begin{array}{l}1.366^{* *} \\
(0.185)\end{array}$ & & $\begin{array}{l}1.584^{* *} \\
(0.315)\end{array}$ & & $\begin{array}{l}1.691^{*} \\
(0.514)\end{array}$ \\
\hline College degree & & $\begin{array}{l}1.785^{* * *} \\
(0.267)\end{array}$ & & $\begin{array}{l}2.034^{* * *} \\
(0.299)\end{array}$ & & $\begin{array}{l}2.062^{* * *} \\
(0.424)\end{array}$ & & $\begin{array}{l}2.569^{* * *} \\
(0.828)\end{array}$ \\
\hline \multicolumn{9}{|c|}{ Importance of religion (Ref: not at all) } \\
\hline Somewhat & & $\begin{array}{l}0.813^{* *} \\
(0.081)\end{array}$ & & $\begin{array}{l}0.779^{* *} \\
(0.099)\end{array}$ & & $\begin{array}{l}0.457^{* * *} \\
(0.059)\end{array}$ & & $\begin{array}{l}0.582^{* *} \\
(0.132)\end{array}$ \\
\hline Quite/very & & $\begin{array}{l}0.410^{* * *} \\
(0.044)\end{array}$ & & $\begin{array}{l}0.402^{* * *} \\
(0.052)\end{array}$ & & $\begin{array}{l}0.247^{* * *} \\
(0.035)\end{array}$ & & $\begin{array}{l}0.141^{* * *} \\
(0.039)\end{array}$ \\
\hline \multicolumn{9}{|c|}{ Working while in college (Ref: never) } \\
\hline Sometimes & & $\begin{array}{l}1.300^{* * *} \\
(0.119)\end{array}$ & & $\begin{array}{l}1.473^{* * *} \\
(0.159)\end{array}$ & & $\begin{array}{l}1.804^{* * *} \\
(0.224)\end{array}$ & & $\begin{array}{l}2.377^{* * *} \\
(0.529)\end{array}$ \\
\hline Regularly & & $\begin{array}{l}1.468^{* * *} \\
(0.189)\end{array}$ & & $\begin{array}{l}1.450^{* *} \\
(0.211)\end{array}$ & & $\begin{array}{l}2.476^{* * *} \\
(0.398)\end{array}$ & & $\begin{array}{l}2.224^{* * * *} \\
(0.666)\end{array}$ \\
\hline In a relationship & & $\begin{array}{l}0.902 \\
(0.077)\end{array}$ & & $\begin{array}{l}1.324^{* * *} \\
(0.124)\end{array}$ & & $\begin{array}{l}1.007 \\
(0.114)\end{array}$ & & $\begin{array}{l}1.472^{* *} \\
(0.288)\end{array}$ \\
\hline \multicolumn{9}{|c|}{ Friends approve sex with persons of same-sex (Ref: No) } \\
\hline Somewhat & & $\begin{array}{l}1.145 \\
(0.135)\end{array}$ & & $\begin{array}{l}1.436^{* *} \\
(0.210)\end{array}$ & & $\begin{array}{l}1.438^{* *} \\
(0.237)\end{array}$ & & $\begin{array}{l}1.089 \\
(0.351)\end{array}$ \\
\hline Quite/yes & & $\begin{array}{l}1.309^{* *} \\
(0.179)\end{array}$ & & $\begin{array}{l}1.629^{* * *} \\
(0.261)\end{array}$ & & $\begin{array}{l}1.776^{* * *} \\
(0.329)\end{array}$ & & $\begin{array}{l}2.290^{* *} \\
(0.756)\end{array}$ \\
\hline \multicolumn{9}{|c|}{ Parents approve sex with persons of same-sex (Ref: No) } \\
\hline Somewhat & & $\begin{array}{l}1.274^{* *} \\
(0.135)\end{array}$ & & $\begin{array}{l}0.994 \\
(0.125)\end{array}$ & & $\begin{array}{l}1.195 \\
(0.168)\end{array}$ & & $\begin{array}{l}0.825 \\
(0.215)\end{array}$ \\
\hline Quite/yes & & $\begin{array}{l}1.059 \\
(0.150)\end{array}$ & & $\begin{array}{l}1.045 \\
(0.164)\end{array}$ & & $\begin{array}{l}1.058 \\
(0.193)\end{array}$ & & $\begin{array}{l}0.686 \\
(0.215)\end{array}$ \\
\hline Constant & $\begin{array}{l}0.207^{* *} \\
(0.129)\end{array}$ & $\begin{array}{l}0.263^{* *} \\
(0.176)\end{array}$ & $\begin{array}{l}0.056^{* * *} \\
(0.041)\end{array}$ & $\begin{array}{l}0.050^{* * *} \\
(0.039)\end{array}$ & $\begin{array}{l}0.211^{*} \\
(0.175)\end{array}$ & $\begin{array}{l}0.423 \\
(0.378)\end{array}$ & $\begin{array}{l}0.038^{* *} \\
(0.060)\end{array}$ & $\begin{array}{l}0.075 \\
(0.127)\end{array}$ \\
\hline N & 3222 & & 2982 & & 3222 & & 2982 & \\
\hline
\end{tabular}

while the hypothesis that bisexual men and women would also have a higher smoking prevalence was only confirmed among women. Our finding that homosexual men and women report a higher smoking prevalence than heterosexual men and women has been consistently observed in the literature (e.g., Lee et al., 2009; Lindley et al., 2012). Further, a recent systematic review and meta-analysis on the bisexual-specific prevalence and likelihood of cigarette smoking found that cigarette smoking was more prevalent among bisexuals than lesbians/gays and heterosexuals, with the estimates showing a greater magnitude among bisexual women relative to all other sexual orientation/gender subgroups (Shokoohi et al., 2020). This comprehensive review supports our finding of the significant difference between bisexual and heterosexual women (and no significant difference among men). 


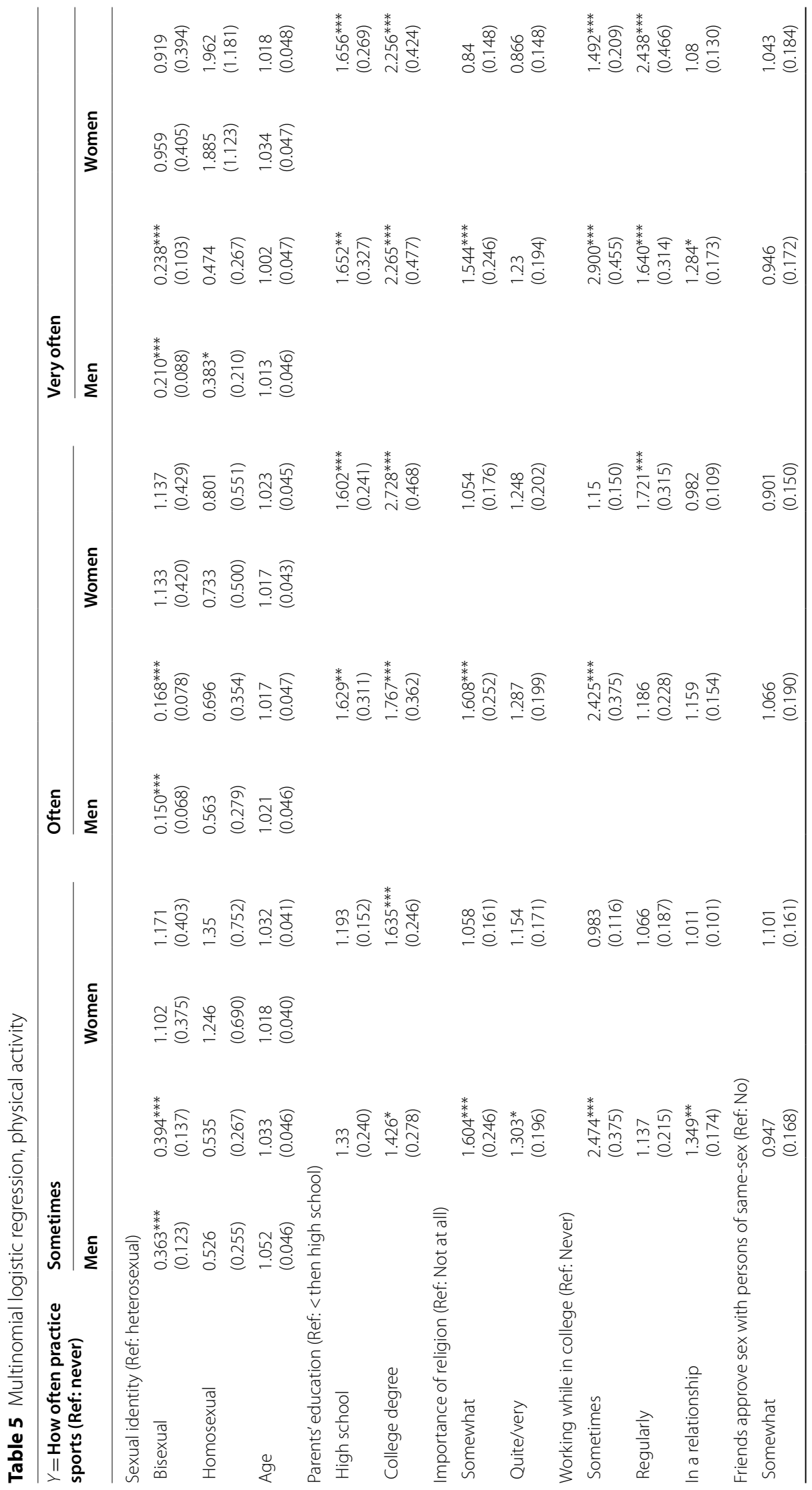




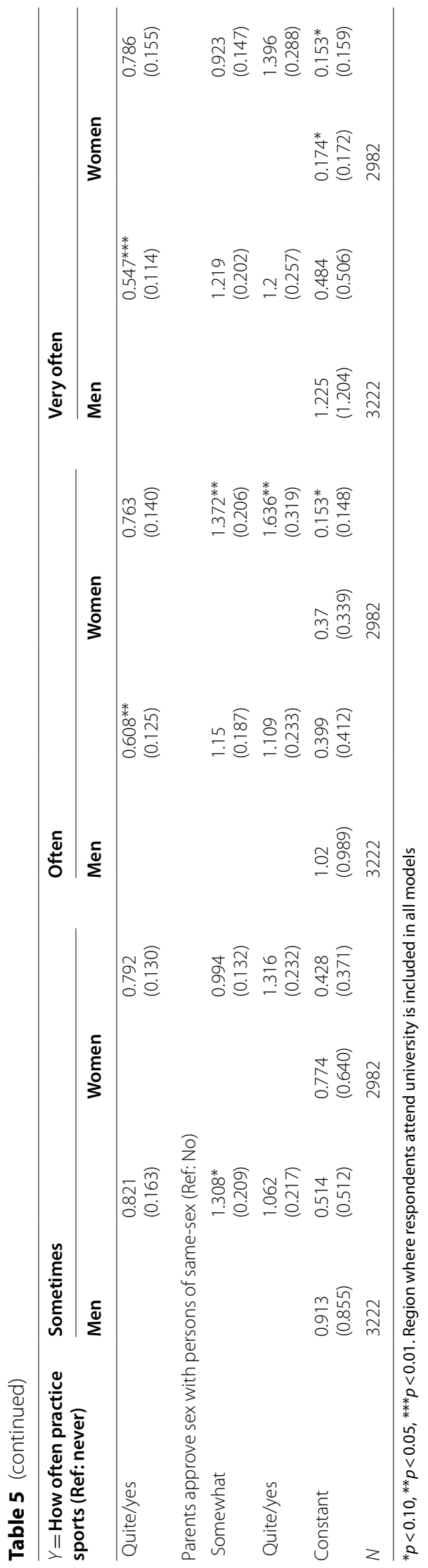


Very similar patterns were found for drinking and marijuana/ecstasy use. Bisexual women were more likely than heterosexual women to get drunk and use marijuana and/or ecstasy sometimes and often/very often, while homosexual women were more likely than heterosexual women to get drunk and use marijuana and/or ecstasy often/ very often. Therefore, our hypotheses that bisexual and homosexual women would report higher drinking and drug use prevalence than heterosexual women were confirmed. Homosexual men were less likely than heterosexual men to get drunk often/ very often, but no additional significant differences among men were observed regarding drinking or marijuana and/or ecstasy use. As such, our hypotheses regarding both drinking and drug use comparing SM and heterosexual men were rejected. Similar results have been found that SM women drink more than heterosexual women, but SM men (depending on dimension) drink less than heterosexual men (Fish, 2019).

And for physical activity, the only significant difference observed was that bisexual men were less likely than heterosexual men to participate in physical activity sometimes, often, and very often. Sexual identity was not significantly associated with physical activity among women. Therefore, only the hypothesis that bisexual men participate in less physical activity than heterosexual men was confirmed. SMs participating in similar or more frequent amounts of physical activity compared to heterosexuals has been observed previously (Fricke et al., 2019). Of note, when the analysis was repeated with changing the reference category to homosexuals, the only significant result was that bisexual men were less likely to exercise often than homosexual men. As such, all of the hypotheses that state that bisexuals would report a higher prevalence of at-risk behaviors than homosexuals were rejected except for physical activity among men. This is in stark contrast with the argument that bisexual individuals embrace risk behaviors to a greater extent than lesbian and gay individuals (D’Augelli et al., 2005).

The only significant differences found between SM and heterosexual men were a higher smoking prevalence among homosexual men and less participation in physical activity among bisexual men. Among women, SM women exhibited a significantly higher prevalence of all of the included at-risk behaviors compared to heterosexual women except for less physical activity. As such, the theories of minority stress, including bi-specific stress, which may be even more relevant in Italy, seem to be more pertinent to the women in this sample. However, bi-specific stress only seems relevant to the bisexual/heterosexual comparison, as bisexuals and homosexuals did not significantly differ (except for men and physical activity). Interestingly, a significantly higher percentage of women than men reported that their friends quite/do approve of sex between persons of the same sex (implying less social stress), but SM women engaged in more at-risk behaviors. For both genders, about $20 \%$ of friends did not approve of sex with the same-sex, while $40 \%$ of parents did not approve. These findings support the aforementioned trends that older people in Italy seem to have more negative attitudes towards SMs than younger people (Baiocco et al., 2013), in addition to males as compared to females (Lingiardi et al., 2016; Santona \& Tognasso, 2018). Gender differences in the perception of and engagement in risky health behaviors have been previously documented (Dawson et al., 2007; Spigner et al., 1993), but further investigation of those differences in the Italian context may be warranted. 
This study was not without limitations. It was cross-sectional, which limits the generalizability of the results. Further, the study did not take into account mental health or other environmental stressors, such as the collegiate environment, which may help explain the observed at-risk behaviors.

\section{Conclusion}

Overall, SM college students in Italy exhibited a higher prevalence of risky health behaviors compared to heterosexuals, especially women. SM women had a significantly higher prevalence of smoking, drinking, and drug use, while SM men only had a higher prevalence of smoking. No significant differences were found between bisexuals and homosexuals except bisexual men exercised less than homosexual men. More attention needs to be given to the identified at-risk populations to inform the Italian national health care system and to ensure that the appropriate care can be provided when required.

Acknowledgements

Not applicable.

Authors' contributions

MS conducted the data analysis and wrote the methods and results sections. JF wrote the background and conclusion sections. Both authors read and approved the final manuscript.

\section{Funding}

No funding was received.

\section{Availability of data and materials}

The datasets used and/or analyzed during the current study are available from the corresponding author on reasonable request.

\section{Declarations}

Competing interests

The authors declare that they have no competing interests.

\section{Author details}

${ }^{1}$ University College London, Gower Street, London WC1E 6BT, UK. ${ }^{2}$ University of Colorado Denver, 1201 Larimer St, Denver, CO 80204, USA.

Received: 10 June 2021 Accepted: 2 October 2021

Published online: 18 October 2021

\section{References}

Baiocco, R., Fontanesi, L., Santamaria, F., loverno, S., Marasco, B., Baumgartner, E., Willoughby, B. L., \& Laghi, F. (2015a). Negative parental responses to coming out and family functioning in a sample of lesbian and gay young adults. Journal of Child and Family Studies, 24(5), 1490-1500. https://doi.org/10.1007/s10826-014-9954-Z

Baiocco, R., loverno, S., Lonigro, A., Baumgartner, E., \& Laghi, F. (2015b). Suicidal ideation among Italian and Spanish young adults: The role of sexual orientation. Archives of Suicide Research, 19(1), 75-88. https://doi.org/10.1080/13811118. 2013.833150

Baiocco, R., Nardelli, N., Pezzuti, L., \& Lingiardi, V. (2013). Attitudes of Italian heterosexual older adults towards lesbian and gay parenting. Sexuality Research and Social Policy, 10(4), 285-292. https://doi.org/10.1007/s13178-013-0129-2

Biddle, S. J. H., \& Mutrie, N. (2008). Psychology of physical activity-determinants well-being and interventions. Routledge.

Bostwick, W., \& Hequembourg, A. (2014). "Just a little hint": Bisexual-specific microaggressions and their connection to epistemic injustices. Culture, Health and Sexuality, 16(5), 488-503. https://doi.org/10.1080/13691058.2014.889754

Caltabiano, M., Castiglioni, M., \& De-Rose, A. (2020). Changes in the sexual behaviour of young people: Introduction. Genus, 76, 38. https://doi.org/10.1186/s41118-020-00107-1

Conron, K. J., Mimiaga, M. J., \& Landers, S. J. (2010). A population-based study of sexual orientation identity and gender differences in adult health. American Journal of Public Health, 100(10), 1953-1960. https://doi.org/10.2105/AJPH. 2009.174169

D'Augelli, A. R., Grossman, A. H., \& Starks, M. T. (2005). Parents' awareness of lesbian, gay, and bisexual youths' sexual orientation. Journal of Marriage and Family, 67(2), 474-482. https://doi.org/10.1111/j.0022-2445.2005.00129.x

Dawson, K. A., Schneider, M. A., Fletcher, P. C., \& Bryden, P. J. (2007). Examining gender differences in the health behaviors of Canadian university students. The Journal of the Royal Society for the Promotion of Health, 127(1), 38-44. 
De Rose, A., Racioppi, F., \& Zanatta, A. L. (2008). Italy: Delayed adaptation of social institutions to changes in family behaviour. Demographic Research, 19, 665-704.

Eisenberg, M., \&Wechsler, H. (2003). Substance use behaviors among college students with same-sex and opposite-sex experience: Results from a national study. Addictive Behaviors, 28(5), 899-913. https://doi.org/10.1016/S03064603(01)00286-6

Fish, J. N. (2019). Sexual orientation-related disparities in high-intensity binge drinking: Findings from a nationally representative sample. LGBT Health, 6(5), 242-249.

Fricke, J., Gordon, N., \& Downing, J. (2019). Sexual orientation disparities in physical activity. Medical Care, 57(2), 138-144.

Frost, D. M., Lehavot, K., \& Meyer, I. H. (2015). Minority stress and physical health among sexual minority individuals. Journal of Behavioral Medicine, 38, 1-8. https://doi.org/10.1007/s10865-013-9523-8

Hsieh, N., \& Ruther, M. (2016). Sexual minority health and health risk factors: Intersection effects of gender, race, and sexual identity. American Journal of Preventive Medicine, 50(6), 746-755. https://doi.org/10.1016/j.amepre.2015.11.016

Ibrahim, A. K., Kelly, S. J., Adams, C. E., \& Glazebrook, C. (2013). A systematic review of studies of depression prevalence in university students. Journal of Psychiatric Research, 47(3), 391-400. https://doi.org/10.1016/j.jpsychires.2012.11.015

Kashubeck-West, S., \& Szymanski, D. M. (2008). Risky sexual behavior in gay and bisexual men. The Counseling Psychologist, 36(4), 595-614. https://doi.org/10.1177/0011000007309633

Kassel, J. D., Stroud, L. R., \& Paronis, C. A. (2003). Smoking, stress, and negative affect: Correlation, causation, and context across stages of smoking. Psychological Bulletin. https://doi.org/10.1037/0033-2909.129.2.270

Lee, J. G., Griffin, G. K., \& Melvin, C. L. (2009). Tobacco use among sexual minorities in the USA, 1987 to May 2007: A systematic review. Tobacco Control, 18(4), 275-282.

Lindley, L. L., Walsemann, K. M., \& Carter, J. W. (2012). The association of sexual orientation measures with young adult's health-related outcomes. American Journal of Public Health, 102(6), 1177-1185. https://doi.org/10.2105/AJPH.2011. 300262

Lingiardi, V., Baiocco, R., \& Nardelli, N. (2012). Measure of internalized sexual stigma for lesbians and gay men: A new scale. Journal of Homosexuality, 59(8), 1191-1210. https://doi.org/10.1080/00918369.2012.712850

Lingiardi, V., Falanga, S., \& D’Augelli, A. R. (2005). The evaluation of homophobia in an Italian sample. Archives of Sexual Behavior, 34(1), 81-93. https://doi.org/10.1007/s10508-005-1002-z

Lingiardi, V., Nardelli, N., loverno, S., Falanga, S., Di Chiacchio, C., Tanzilli, A., \& Baiocco, R. (2016). Homonegativity in Italy. Sexuality Research and Social Policy, 13(2), 95-108.

Mereish, E. H. (2014). The weight of discrimination: The relationship between heterosexist discrimination and obesity among lesbian women. Psychology of Sexual Orientation and Gender Diversity, 1(4), 356-360. https://doi.org/10.1037/ sgd0000056

Mereish, E. H., Katz-Wise, S. L., \& Woulfe, J. (2017a). Bisexual-specific minority stressors, psychological distress, and suicidality in bisexual individuals: The mediating role of loneliness. Prevention Science, 18(6), 716-725. https://doi.org/10. 1007/s11121-017-0804-2

Mereish, E. H., Katz-Wise, S. L., \& Woulfe, J. (2017b). We're here and We're queer: Sexual orientation and sexual fluidity differences between bisexual and queer women. Journal of Bisexuality, 17(1), 125-139. https://doi.org/10.1080/15299 716.2016 .1217448

Meyer, I. H. (2003). Prejudice, social stress, and mental health in lesbian, gay, and bisexual populations: Conceptual issues and research evidence. Psychological Bulletin, 129(5), 674-697. https://doi.org/10.1037/0033-2909.129.5.674

Meyer, I. H., \& Northridge, M. E. (2007). The health of sexual minorities: Public health perspectives on lesbian, gay, bisexual, and transgender populations. Springer. https://doi.org/10.1007/978-0-387-31334-4

Minello, A., Caltabiano, M., Dalla-Zuanna, G., \&Vignoli, D. (2020). Catching up! The sexual behaviour and opinions of Italian students (2000-2017). Genus, 76(1), 1-22.

Mohr, J. J., \& Daly, C. A. (2008). Sexual minority stress and changes in relationship quality in same-sex couples. Journal of Social and Personal Relationships, 25(6), 989-1007. https://doi.org/10.1177/0265407508100311

Newcomb, M. E., Heinz, A. J., \& Mustanski, B. (2012). Examining risk and protective factors for alcohol use in lesbian, gay, bisexual, and transgender youth: A longitudinal multilevel analysis. Journal of Studies on Alcohol and Drugs, 73(5), 783-793. https://doi.org/10.15288/jsad.2012.73.783

Pelullo, C. P., Di Giuseppe, G., \& Angelillo, I. F. (2013). Frequency of discrimination, harassment, and violence in lesbian, gay men, and bisexual in Italy. PLOS ONE, 8(8), e74446. https://doi.org/10.1371/journal.pone.0074446

Petruccelli, I., Baiocco, R., loverno, S., Pistella, J., \& D'Urso, G. (2015). Possible families: A study on attitudes toward same-sex family. Giornale Italiano Di Psicologia, 42(4), 805-828. https://doi.org/10.1421/81943

Regier, D., Farmer, M. E., Rae, D. S., Locke, B. Z., Keith, S. J., Judd, L. L., \& Goodwin, F. (1990). Comorbidity of mental disorders with alcohol and other drug abuse. Results from the Epidemiologic Catchment Area (ECA) Study. JAMA, 264(19), 2511-2518. https://doi.org/10.1001/jama.1990.03450190043026

Salvati, M., Pistella, J., loverno, S., Giacomantonio, M., \& Baiocco, R. (2018). Attitude of Italian Gay Men and Italian lesbian women towards gay and lesbian gender-typed scenarios. Sexuality Research and Social Policy, 15(3), 312-328. https:// doi.org/10.1007/s13178-017-0296-7

Santona, A., \& Tognasso, G. (2018). Attitudes toward homosexuality in adolescence: An Italian study. Journal of Homosexuality, 65(3), 361-378. https://doi.org/10.1080/00918369.2017.1320165

Shokoohi, M., Salway, T., Ahn, B., \& Ross, L. E. (2020). Disparities in the prevalence of cigarette smoking among bisexual people: A systematic review, meta-analysis and meta-regression. Tobacco Control. https://doi.org/10.1136/tobac cocontrol-2020-055747

Spigner, C., Hawkins, W. E., \& Loren, W. (1993). Gender differences in perception of risk associated with alcohol and drug use among college students. Women \& Health, 20(1), 87-97.

VanKim, N. A., Bryn, A. S., Hee-Jin, J., \& Corliss, H. L. (2017). Physical activity and sedentary behaviors among lesbian, bisexual, and heterosexual women: findings from the nurses' health study II. Journal of Women's Health. https://doi. org/10.1089/jwh.2017.6389

Vignoli, D., \& Salvini, S. (2014). Religion and union formation in Italy: Catholic precepts, social pressure, and tradition. Demographic Research, 31, 1079-1106. 
Worthen, M. G. F. (2013). An argument for separate analyses of attitudes toward lesbian, gay, bisexual men, bisexual women, MtF and FtM transgender individuals. Sex Roles, 68(11-12), 703-723. https://doi.org/10.1007/ s11199-012-0155-1

Worthen, M. G. F., Lingiardi, V., \& Caristo, C. (2017). The roles of politics, feminism, and religion in attitudes toward LGBT individuals: a cross-cultural study of college students in the USA, Italy, and Spain. Sexuality Research and Social Policy, 14(3), 241-258. https://doi.org/10.1007/s13178-016-0244-y

\section{Publisher's Note}

Springer Nature remains neutral with regard to jurisdictional claims in published maps and institutional affiliations.

Submit your manuscript to a SpringerOpen ${ }^{\circ}$ journal and benefit from:

- Convenient online submission

- Rigorous peer review

- Open access: articles freely available online

- High visibility within the field

- Retaining the copyright to your article

Submit your next manuscript at $\gg$ springeropen.com 